\title{
Investigar-pensar desde los Estados del Arte: Punto de partida Plantear problemas
}

\author{
Research-Thinking from the States of Art: Starting Point Posing Problems
}

Dra: Rosaura Castillo Guzmán

Dra. Angelica Maria Fabila Echauri

Dra. Flor de Liz Pérez Morales

\section{RESUMEN}

La investigación y el pensamiento como dualidad, deben ser consideradas como fuente para la creación de ideas para ser investigadas; lo anterior recobra fuerza cuando reconocemos que tan importante es enseñar a investigar a los estudiantes universitarios, lo que implica entonces que alguien debe hacer esa tarea primordial.

En las aulas quien enseña a investigar es el docente responsable de aquellas asignaturas que, desde el currículo, marcan esa encomienda; hecho que parece oponerse a la premisa que en más de una ocasión hemos escuchado: se enseñanza a investigar investigando. La incertidumbre del encuentro entre docente-experto y aprendiz-novato en el aula para enseñar y aprender a investigar es una de las discusiones que permiten reflexionar que, si bien existen argumentos para sostener que se enseña a investigar investigando, es igualmente válido considerar que enseñar a pensar una idea para investigar y elevarla a la categoría de problema, se puede hacer desde el aula.

Desde la óptica de las autoras, una estrategia para enseñar y aprender a investigar pueden ser los estados del arte, ya que el encuentro que el aprendiz-novato tiene con las ideas de otros, que ante su mirada son investigadores-expertos, genera la posibilidad de activar su pensamiento y abrir perspectivas alternas que le conduzcan a superar la incertidumbre que presenta siempre el asunto a investigar, ampliando así la experiencia que tiene como referente para hacer investigación. Pensar una idea es una tarea que debe colocarse en el centro de quien enseña a investigar en el aula.

La intención del presente reporte es colocar en el centro de la discusión algunos ejes encontrados en la investigación sobre la construcción del estado del arte 0 del conocimiento, de las habilidades de pensamiento y de investigación que desarrollan los estudiantes a partir de la construcción de los estados del arte, y como estas habilidades posibilitan al estudiante a plantear problemas de investigación.

Palabras claves: habilidades investigativas, habilidades de pensamiento, enseñar a pensar-investigar.

\section{ABSTRACT}

Research and thought as duality, must be considered as a source for an idea creation to be investigated. This regains strenght when we recognize how important it's to teach University students to investigate, which implies someone must do that important task.

In the classroom, the responsible teacher of those subjects is the main one to teach to investigate; that's why it's asked in the curriculum; a fact that seems to oppose the premise that maybe we've heard more than once: it's taught to investigate, investigating! The uncertainty of the meeting among teacher-expert and student-learner in the classroom, at the moment to teach and learn to investigate is one of the matters that allow us to reflect about: if there are some arguments about it's taught to investigate investigating, so it's valid to consider too that teaching to think is a good idea to investigate; and getting it to the level of the problem can be surely done from the classroom.

One strategy to teach and learn to investigate may be the states of art, where it's possible that the student-learner activates his thinking in a better way at the moment to be face to face with the ideas from another people, such as "expert researchers" and finally resorts to the experience gotten already as a reference to start the research. Thinking about an idea is a task that must be considered as the primary one, who teach to investigate in the classroom.

The intention of this report is to place in the center of the discussion some basis found in the investigation about the construction of the state of art and knowledge, the skills of awareness 
Perspectivas Docentes 64

Investigar-pensar desde los Estados del Arte: Punto de partida Plantear problemas

Research-Thinking from the States of Art: Starting Point Posing Problems

Dra: Rosaura Castillo Guzmán / Dra. Angelica Maria Fabila Echauri / Dra. Flor de Liz Pérez Morales

and investigation that develop the students from the construction of the state of art, and how these skills enable the student to solve research problems.

Keywords: Research ability, Ability of thought, Teach to think-research.

\section{INTRODUCCIÓN}

Abordar la temática de los estados del arte y el aprendizaje desde el contexto de la formación inicial en investigación en el marco de las actividades sustantivas de las universidades, encuentra su fundamento en el paradigma de la complejidad, articulado con el enfoque constructivista. Dar claridad al asunto de las habilidades que posee el estudiante y la importancia de movilizarlas en un proceso de construcción y de aprendizaje, es el punto de reflexión de este ensayo, que tiene como referente la investigación denominada Estados del arte y/o del conocimiento: Aproximaciones a las formas de aprendizaje del estudiante en el proceso de investigación inicial, teniendo como sujetos de estudio a estudiantes que cursaron la asignatura de Metodología, en la Universidad Juárez Autónoma de Tabasco, de la División Académica de Educación y Artes. El objetivo general fue comprender qué habilidades de pensamiento y de investigación fueron desarrolladas por el estudiante en la construcción del estado del arte, reconociendo las posibilidades que hay de enseñarlas en el aula; la investigación consideró algunos objetivos específicos como el identificar el procedimiento metodológico utilizado por el estudiante en la construcción del estado del arte o del conocimiento, reconocer la habilidad de pensamiento que prevalece en el estudiante para generar la idea inicial de investigación.

La enseñanza de la investigación está determinada desde el curriculum universitario. Al respecto Jiménez, Moreno y Ortiz (2011) expresan que la formación y desarrollo de investigadores se sigue realizando principalmente en las instituciones de educación superior, especialmente las universidades públicas, siendo éstas las que se constituyen en el principal punto de partida de formación para los nóveles investigadores.

Es necesario insistir que los estudiantes pueden acceder al conocimiento de la realidad a través de la investigación, es importante considerar lo que plantea el paradigma del pensamiento complejo, en cuanto a provocar en el estudiante incertidumbre ante la misma realidad para que actúe de acuerdo a las circunstancias del contexto, la actuación que se espera de ellos es la intención de acercarse a través de la investigación.

El docente-experto debe orientar al estudiante (aprendiz-novato) para que descubra sus propias formas de aprender y generar conocimiento, esta debe ser, una de las metas educativas y pedagógicas de la práctica docente. Al respecto Morín (1996), hace referencia que cada vez que un profesor enseña algo a sus alumnos, el contenido de lo que comunica se plantea a manera de un determinado sistema de representación, y esto ocurre en cada experimento 0 actividad de información, lo cual se organiza con ciertos puntos de vista dominantes 0 paradigmas, que a menudo son versiones simplificadas de realidades complejas.

La enseñanza de los estados del arte, la manera particular de recuperar experiencias desde aprendiz de su propio proceso didáctico, y el aprender a investigar, debe permitir al novato a acceder a la realidad, reconociendo que esta es cambiante, incierta incluso compleja que requiere de sujetos con capacidad para adaptarse, para pensar y responder a las exigencias del mundo.

Una de las primeras opciones que se les puede mostrar a los estudiantes son los planteamientos o resultados de investigación de expertos que han explicado el mundo desde perspectivas diferentes, siguiendo caminos metodológicos diversos, para que de ahí parta a la confrontación de ideas y estar en posibilidades de proponer otras rutas desde donde pueden ser investigadas. Así, enseñar a pensar es una de las mejores opciones para iniciarse en la investigación, y en ese marco de posibilidades la estrategia de aprender a aprender a través de los estados del arte cobra un papel importante.

Para Blanco (2010), la investigación está ligada al pensamiento, por lo que investigar es una actividad que puede ser practicada por todo aquel que siga las exigencias básicas del pensamiento riguroso; no es una tarea que excluya, singularice y distinga a las personas que quieren conocer 0 mejorar las áreas de conocimiento. Ser investigador es, antes que nada, ser estudioso de algo, alguien que piensa, que busca conocimiento. Investigar es básicamente pensar.

De igual forma Blanco expone que el pensamiento no siempre es lo necesariamente preciso, riguroso, sistemático; ni está siempre sujeto a un plan, guiado por un método o sometido a 
Perspectivas Docentes 64

Investigar-pensar desde los Estados del Arte: Punto de partida Plantear problemas

Research-Thinking from the States of Art: Starting Point Posing Problems

Dra: Rosaura Castillo Guzmán / Dra. Angelica Maria Fabila Echauri / Dra. Flor de Liz Pérez Morales

crítica y revisión...en término académico se suele reconocer como la investigación no siempre se acomoda a las normas o criterios de calidad del pensamiento riguroso.

En este sentido, si investigar requiere del pensamiento, en necesario ayudar al novato a pensar para investigar, es decir dirigir el pensamiento a un objeto de interés, para que cree una idea y le dé forma. En el proceso de indagación la idea debe estar relacionada con el objeto de estudio que se desea conocer o profundizar, lo que posibilitara una comprensión diferente; permitirá encaminar al pensamiento hacia lo que Edgar Morín llama pensamiento complejo, es decir, pasar de la simplicidad de las cosas hasta llegar a hacer rupturas que impliquen nuevas respuestas. Provocar la crisis de conocimiento generada por la incertidumbre del pensamiento, para encontrar relaciones estrechas en un contexto general, en la vida misma, y con apoyo en diversas disciplinas explicar la realidad.

Lo anterior, exige terminar con la inercia mental en la que muchos estudiantes caen al momento de tener que pensar en una idea de investigación, al momento de plantear un problema, Martínez (1997) en su texto el Paradigma Emergente, recupera algunos señalamientos de Kant sobre la génesis del conocimiento, donde expone que el factor activo no es el objeto sino el sujeto, no son las cosas sino la mente la que lleva por dentro toda la ciencia potencial y actual.

Martínez continúa recuperando a Kant en lo referente a que la mente humana es un participante activo o formativo de lo que ella conoce, la mente construye y el entendimiento humano se convierte en un constructor de su propio mundo.

Podemos expresar entonces, que el desarrollo de las habilidades cognitivas no es una tarea pedagógica nueva, el desarrollo de la capacidad de pensar, de conocer, es un replanteamiento necesario en los escenarios pedagógicos actuales, y no sólo es la capacidad de pensar, sino de enseñar a pensar en las partes y en el todo de aquello que se está pensando investigar.

Lo anterior puede encontrar un sustento en lo expuesto por Martínez (1997), cuando expresa que pensar es la suprema función del hombre, y a través de este ejercicio resuelve problemas que la vida le plantea. Pero, para que la mente trabaje en forma eficiente, necesita una buena dosis de intenso aprendizaje. Reconocer que la dinámica mental juega un papel en la búsqueda, selección y descubrimiento de diferentes tipos de contenidos, incluso la construcción de nuevos significados.

\section{DESARROLLO}

El estudio que se presenta es parte de una exploración realizada en estudiantes universitarios de la División Académica de Educación y Artes, de la Universidad Juárez Autónoma de Tabasco, que cursaron la asignatura de Metodología; mismo que dio sustento al trabajo ya referido y denominado Estados del arte y/o del conocimiento: Aproximaciones a las formas de Aprendizaje del estudiante en el proceso de investigación inicial.

Se planteó en el inicio del presente ensayo, la necesidad de comprender que habilidades de pensamiento y de investigación son desarrolladas por el estudiante en la construcción del estado del conocimiento, para ello, Identificar qué están aprendiendo los estudiantes y cómo lo están haciendo es una tarea que debe realizar el profesor-investigador que enseña a investigar en el aula.

La recomendación de enseñar a investigar investigando implica comprometer al experto a guiar al estudiante-novato para que logre aproximarse al conocimiento. Esto se construye a partir de una autorreflexión de cómo aprendió a hacer estados del arte, para ello, debe explorar en la propia experiencia de cómo aprendió a hacerlo y de cómo enseña lo aprendido. En este sentido, Morín pone énfasis en la idea que el docente debe ser el primero en tomar conciencia de su papel y posición de influencia para alcanzar la meta de una educación que aspire a una formación integral (Cabrera, 2014 p.79).

Desde esta perspectiva, el objeto de estudio de la investigación justifica la necesidad de reconocer en el novato, procesos de aprendizaje y de pensamiento que se activan a partir de la aplicación de la propuesta metodológica, como es hacer uso de los estados del arte, como parte de un proceso para el inicio en la investigación, con ello, lograr el inicio del estudiante en la investigación, mientras debe reconocerse que el experto es quien guía al novato en el proceso de construcción, hasta lograr que el estudiante plantee el problema.

El trabajo de campo permitió la recuperación de experiencias vividas por los estudiantes, relacionar estas experiencias con algunos referentes conceptuales, además de la comprensión de que los estados del arte como metodología para aproximarse 
Perspectivas Docentes 64

Investigar-pensar desde los Estados del Arte: Punto de partida Plantear problemas

Research-Thinking from the States of Art: Starting Point Posing Problems

Dra: Rosaura Castillo Guzmán / Dra. Angelica Maria Fabila Echauri / Dra. Flor de Liz Pérez Morales

al planteamiento del problema, son una opción compleja, pero que guiado por un docente-experto logren desarrollarla.

Lo expresado por los sujetos que conformaron el grupo de informantes dotaron de fundamento y sentido a la investigación sobre los estados del arte, especialmente en relación a cómo se aprende a través de esta metodología o estrategia de enseñar y aprender, y con ello estar en posibilidades para dar paso a la formación inicial en investigación.

Las respuestas de los informantes develaron esquemas de pensamiento y potenciales habilidades investigativas que los estudiantes poseen y que necesitan ser desarrolladas con estrategias en el aula.

Enseñar a investigar con la propuesta sugerida amplia el estudio, al describir el proceso que se activa en el sujeto al momento de elaborar estados del arte; de la misma manera, permitió identificar qué sucede en términos de aprendizaje, de desarrollo de habilidades y de procedimientos desarrollados en la construcción de saberes, cuando el estudiante se acerca a la investigación a través de la propuesta mencionada, además de constituirse como una estrategia de aprendizaje activa, es decir, un aprender investigando.

Lo anterior, encuentra fundamento en la propuesta de Rojas (2007), sobre las fases en las que se desarrolla el estado del arte, por un lado la fase hermenéutica o de descubrimiento, que implica la búsqueda y recopilación de las fuentes de información (estas se pueden considerar habilidades investigativas), por otro lado, la fase hermenéutica, donde quien realiza esta búsqueda debe echar mano de algunas habilidades que tiene que ver con el pensamiento, y que al ponerlas en práctica está desarrollando habilidades cognitivas que puede llevar al novato al aprendizaje.

La pertinencia de este objeto de estudio, posibilita el acceso a las experiencias vividas por los estudiantes-novatos con respecto a la elaboración de un estado del arte, las experiencias compartidas y recuperadas develan cuáles son las habilidades cognitivas o habilidades de construcción que se activan durante el proceso de elaboración de las formas de acceso al proceso de investigación, y cómo van resolviendo sus necesidades de investigación.

El reconocimiento del proceso metacognitivo que utiliza el estudiante en un proceso de aprendizaje en investigación inicial favorece la construcción de conocimiento. Al respecto, Bueno
(2003) considera que la actividad cognitiva y metacognitiva en el campo de la investigación o fuera de ella, no solamente está formada por operaciones con las representaciones sensibles y los conceptos, sino que con las cosas mismas desempeña relevante papel; la actividad presupone la utilización de representaciones, conceptos, construcciones lógicas y lingüísticas, y otros elementos que caracterizan la conciencia dirigida hacia un fin (p.10).

Es necesario que los programas de formación inicial en investigación, recuperen la dualidad de experto-novicio (novato), donde el papel del docente se defina como el de un "acompañante cognitivo" que oriente las actividades cognitivas requeridas en el proceso de investigación que realiza el estudiante, señala Tedesco (2003) que en un aprendizaje escolar los procedimientos que utiliza el estudiante están ocultos y el docente debe ser capaz de exteriorizar el proceso mental, de tal manera que el alumno logre observar y comparar a partir de su propio modo de pensar.

Hacer que el estudiante tenga el control de su vida mental y la autorregulación de sus facultades cognitivas hará posible el aprendizaje, con ello, la planificación de una actuación inteligente, desde la perspectiva de Tesouro esto es metacognición.

Con base a lo anterior se delinearon las cuatro categorías centrales exploradas en los investigadores novatos que participaron en el estudio: estados del arte, habilidades metacognitivas (o de pensamiento), habilidades de investigación y habilidades cognitivas.

\section{LAS CATEGORÍAS DE ESTUDIO:}

Derivadas de las cuatro categorías centrales del estudio se desprenden los ejes de análisis o subcategorías, las cuales durante la recuperación y organización de la información proporcionada por los sujetos de estudios encontraron una explicación.

1.- Estados del arte:

- Utilidad como estrategia-recurso

- Dificultades de uso

- Procedimiento de elaboración

2.- Habilidades metacognitivas o de pensamiento 
Perspectivas Docentes 64

Investigar-pensar desde los Estados del Arte: Punto de partida Plantear problemas

Research-Thinking from the States of Art: Starting Point Posing Problems

Dra: Rosaura Castillo Guzmán / Dra. Angelica Maria Fabila Echauri / Dra. Flor de Liz Pérez Morales

- Pensamientos

- Generación de idea de investigación

3.- Habilidades Investigativas

- Búsqueda de información

- Idea de investigación

- Definición de objeto de estudio

4.- Habilidades cognitivas

- Memoria

- Asociación

- Discriminación

El enfoque referente desde donde se visualiza el objeto de interés, es el estudio cualitativo, para Sandín (2004) la intención de este enfoque, es lograr la comprensión holística del objeto de estudio que se observó, además permite tener un acercamiento detallado de situaciones, eventos, personas, interacciones o comportamientos que fueron observables, incorporando la voz de los participantes. La postura metodológica que permitió la integración de los fundamentos para alcanzar la comprensión sobre el uso de los estados del arte como estrategia de aprendizaje para la investigación, según la voz de los protagonistas, y el registro de experiencias, nos ayudó a reconocer las formas de aproximación que logró el novato al objeto de estudio, con ello, la identificación de las habilidades cognitivas e investigativas movilizadas en el proceso de construcción, y la manera cómo aprende el estudiante-novato en su inicio de la investigación.

La formación inicial de investigadores, en este caso de los estudiantes-novatos, requiere encontrar las formas apropiadas y crear las condiciones necesarias, para que éste acceda al conocimiento a través de sus propias experiencias de aprendizaje; para reconocer las experiencias de aprendizaje que tienen los estudiantes a partir de su incorporación a procesos de investigación inicial, el estudio exploratorio fue la opción pertinente para acceder a los sujetos que conformaron la muestra de esta investigación. De acuerdo con Babbie (2000) este tipo de estudios son para realizar exploraciones sobre un tema o para familiarizarnos con alguna cuestión. El planteamiento habitual para los estudios exploratorios, es cuando un investigador examina un nuevo interés o cuando el objeto de estudio es, en sí, relativamente nuevo.

Los estudios exploratorios también son apropiados, según Babbie, para fenómenos más persistentes. Por ejemplo, con respecto al objeto de estudio abordado en el trabajo de investigación sobre elaboración de estados del arte, las habilidades investigativas y de pensamiento se eligieron como objeto de indagación, ya que en los últimos años algunos docentes que enseñanza a investigar en las universidades y en otras instituciones de educación superior, sugieren la construcción del estado del arte como un primer paso para iniciar trabajos de investigación, o iniciar a los estudiantes en una formación previa en investigación.

Para sustentar esta idea persistente sobre la formación inicial, fue necesario recurrir a aquellos estudiantes-novatos (estudiantes de licenciatura) que han investigado usando la estrategia mencionada, con ello describir el procedimiento metodológico que utilizan en su construcción, cómo dan forma a la idea inicial de investigación al momento de pensar y confrontar esta con lo encontrado en el ejercicio del estado del arte, la investigación de igual forma contribuyo en la identificación de aquellas habilidades metacognitivas y cognitivas utilizadas en el proceso de aprender a aprender investigando.

Muchas veces la investigación exploratoria se efectúa con grupos focales o de interés, o discusiones guiadas de grupos pequeños, apoyada por algún instrumento de recolección de información, como es el caso de preguntas guías que permitan al sujeto ofrecer información para poder identificar a partir de los datos, si ellos reconocen las formas que acceder al conocimiento, de las formas como realizan sus procesos de aprendizaje al momento de hacer investigaciones o elaborar estados del arte, de igual forma la sugerencia de iniciarse 0 lograr una formación inicial en investigación.

\section{METODOLOGÍA DEL ESTUDIO:}

En una investigación, es importante la elección de la muestra, por su relevancia en la construcción de datos, elegir a los sujetos clave es tener acceso a su experiencia desde la realidad comprendida por éstos, quienes comparten datos significativos al trabajo, información que deben ser interpretadas con objetividad. La muestra desde la percepción de Babbie (2000) es en cada caso escoger una parte de los datos que podríamos recopilar y estudiar.

Desde luego, el muestreo de la información ocurre en la vida diaria y a menudo produce observaciones, en este caso los estudiantes elegidos permitieron con su información generar 
Perspectivas Docentes 64

Investigar-pensar desde los Estados del Arte: Punto de partida Plantear problemas

Research-Thinking from the States of Art: Starting Point Posing Problems

Dra: Rosaura Castillo Guzmán / Dra. Angelica Maria Fabila Echauri / Dra. Flor de Liz Pérez Morales

datos para ser analizados, y de esta manera explicar bajo qué fundamento guían su actuación en el campo de la investigación a partir de un estado del arte. Cabe destacar que un investigador de campo necesitaría sólo a los informantes que le den una imagen equilibrada de la situación que estudia, y un experimentador asignaría los sujetos a los grupos experimental y de control de forma tal que fueran comparables (Babbie 2000).

El proceso de selección de la muestra de este estudio (sujetos clave), estuvo sujeta a ciertas características de los informantes: ser estudiantes universitarios y haber tenido la experiencia de la aplicación del estado del arte como parte de su formación inicial. Para el caso fueron seleccionados dos grupos de estudiantes de diferentes ciclos cursados.

Bajo estos referentes metodológicos, el trabajo de campo se desarrolló en dos fases: la primera fase consideró a estudiantes inscritos en la asignatura de Metodología en el ciclo corto febrero-julio 2012, la oferta fue bajo la modalidad de movilidad, lo que permitió contar con estudiantes que procedían de distintas licenciaturas (1 Nutrición, 2 Idiomas, 2 Ciencias de la Educación, 3 Contaduría, 1 Historia), los estudiantes inscritos cursaban esta asignatura del área general con créditos obligatorios.

La mayoría de los planes de estudios de la Universidad Juárez Autónoma de Tabasco ubican curricularmente esta asignatura entre segundo y tercer ciclo. Con este grupo de estudiantesnovatos se aplicó la técnica grupo de discusión, técnica donde la apertura lograda por los participantes recreó la experiencia su aprendizaje frente a los estados del arte.

El grupo de discusión es una técnica que de acuerdo con Delgado y Gutiérrez (2003) permite detectar a través del discurso grupal cómo aprendieron a investigar, qué habilidades desarrollaron en el ámbito del pensamiento, la metacognición y de las propias habilidades de la investigación. La ventaja de esta técnica es que se genera una conversación entre iguales y al darse el proceso de intervención de individualidades lo que se pretende es interconectar puntos de vista y, desde la mirada de los participantes describir con objetividad lo referente al objeto de estudio.

Otra ventaja del uso de la técnica fue, detonar la memoria de los participantes, considerada por Aguirre Lora en su texto Rostros históricos de la educación (2001) como un recurso para reconstruir la historia, y traer al presente aspectos relevantes vividos. En este caso los participantes no solo recordaron los pasajes más significativos de haber trabajo estados del arte como estrategia para generar ideas, sino detonaron procesos de pensamiento 0 de reflexión de la memoria individual frente a una memoria colectiva que ofreció a cada novato a una exposición de significados particulares. Ello significa que al momento de producirse a sí mismo como una entidad discursiva, puede decirse que el discurso que van produciendo se constituye en un instrumento para pensar el pensamiento (Ibáñez, 1994).

La segunda fase de este trabajo de campo, se conformó con la selección de un segundo grupo con estudiantes de la licenciatura en idiomas del ciclo escolar agosto 2012-enero 2013, a quienes se les aplicó un cuestionario, el propósito recuperar de "viva voz" su experiencia de aprendizaje a través del estado del arte, y la valoración que le dan a esta estrategia de investigación como parte de un proceso de formación inicial en investigación.

El diseño del cuestionario tuvo como referente la información recuperada del grupo de discusión de los primeros informantes, de los datos se elaboraron las preguntas con la intención de que las categorías fueran las mismas; se aplicó un cuestionario con preguntas abiertas a 12 estudiantes de tercer ciclo que cursaban la asignatura de metodología, de los cuales se recuperaron siete cuestionarios (en este ensayo se presenta una matriz como ejemplo, contiene tres de los siete cuestionarios, el análisis corresponde a las siete muestra recuperadas)

Si bien es cierto los cuestionarios se asocian con los enfoques y diseños cuantitativos, para fines de esta investigación con diseño cualitativo, el uso del cuestionario se fundamenta bajo el argumento de Rodríguez et. al. (1996), señalan que para su elaboración y administración debe considerar algunas exigencias fundamentales, tales como:

- El cuestionario es un procedimiento de exploración de ideas y creencias generales sobre algún aspecto de la realidad;

- El cuestionario se considera una técnica más, no la única ni la fundamental, en el desarrollo del proceso de recogida de datos: 
Perspectivas Docentes 64

Investigar-pensar desde los Estados del Arte: Punto de partida Plantear problemas

Research-Thinking from the States of Art: Starting Point Posing Problems

Dra: Rosaura Castillo Guzmán / Dra. Angelica Maria Fabila Echauri / Dra. Flor de Liz Pérez Morales

- En análisis de los datos del cuestionario permite que la información se comparta por participantes en la investigación.

De acuerdo con Rodríguez et. al. (1996) la forma que adopta el cuestionario debe entenderse como una traducción 0 concreción de los supuestos, creencias o modelos de partida para explicar una realidad. Es decir, las preguntas que figuran en este tipo de técnicas reflejan lo que se piensa acerca del problema que se está investigando. La intención del cuestionario aplicado a los estudiantes fue explorar sobre la experiencia que tuvieron al utilizar la metodología del estado del arte como estrategia para aprender a investigar a aprender a pensar, y de manera puntual para plantear problemas, las respuestas que se obtienen se explican desde el esquema conceptual que fundamenta teóricamente este trabajo.

La información recuperada de las técnicas aplicadas, ofrecen datos que fueron triangulados para analizar la diversidad de explicaciones a partir de un esquema de categorías definidas con anterioridad e incluidas en las preguntas guías del cuestionario utilizado.

\section{ALGUNOS RESULTADOS:}

La triangulación para Denzi en Rodríguez (2010), "es una técnica para incrementar la validez de los resultados de una investigación mediante la depuración de las deficiencias intrínsecas de un solo método de recogida de datos y del control del sesgo personal de los investigadores. De este modo puede decirse que cuanto mayor es el grado de triangulación, mayor es la fiabilidad de las conclusiones alcanzadas".

La triangulación de la información, es una acción que se lleva a cabo una vez que se ha concluido con la recopilación a través de los instrumentos seleccionados, en este caso, lo videograbado en el grupo de discusión y el cuestionario a estudiantes, los instrumentos aplicados cada uno de los grupos de estudiantes, estuvieron diseñados con una lógica centrada en las categorías y subcategorías de estudio lo que permitió establecer una relación conceptual entre ellas; con el uso de estas impone buscar entre la información recuperada, lo que cada idea o respuesta tiene en común con los otros aportes, en el contexto del manejo de los datos permite agrupar las partes que tienen en común entre sí.
Para presentar los resultados señala González (2003) se hace necesario hacer una sistematización y un análisis de los datos recuperados, para ello, se recurre a la inducción como método de razonamiento riguroso que sistematiza cada uno de los datos que se generan al obtenerse de los sujetos informantes.

Las particularidades evidenciadas en el grupo de discusión y en los cuestionarios, se convierten previa organización en categorías que son "llenadas" con explicaciones recuperadas, debe permitir de manera objetiva hacer una generalizada, comprensión del objeto de estudio.

Con respecto al grupo de discusión, se revisó la videograbación, el foco de atención estuvieron en las respuestas dadas por los participantes a partir de las preguntas guías, para organizar la información se identificaron y definieron categorías, porque fue necesario dar significado a los resultados, ya que al ser elementos básicos permitieron hallar explicaciones a partir de lo que se recogió y se organizó de la información proporcionada, contrastada con las teóricas que dan fundamento al objeto de estudio.

En lo referente a las respuestas de los cuestionarios, el ejercicio de organización de la matriz, tuvo como punto de referencia la idea que produce cada pregunta, y que fue explicada por escrito por el participante, es decir por el novato que tuvo la experiencia de elaborar un estado del arte, como fase de inicio en investigación, que lo llevo posteriormente al planteamiento de un problema.

La representación gráfica de la matriz, busca contextualizar las nociones generadas por los estudiantes, describirlas y especificar las relaciones conceptuales o de ideas que lograron explicarse a partir de las categorías y subcategorías que conformaron el instrumento de recuperación de información, cabe mencionar que se encontraron otros rasgos que se integraron a las categorías y subcategorías existentes, así como la revisión de la información recuperada no develo datos para explicar algunas categorías y subcategorías definidas desde el inicio de la investigación.

Las categorías hay que recordar, se utilizan para dar orden conceptual a los datos y poder presentar la información por el contenido y su interpretación, además permiten ordenar la variedad de ideas o conceptos generados en la conversación o en el cuestionario. Un dato necesario de aclarar, es que la matriz muestra las repuestas dadas por los participantes, algunas categorías agrupan pocas respuestas, sin embargo, 
Perspectivas Docentes 64

Investigar-pensar desde los Estados del Arte: Punto de partida Plantear problemas

Research-Thinking from the States of Art: Starting Point Posing Problems

Dra: Rosaura Castillo Guzmán / Dra. Angelica Maria Fabila Echauri / Dra. Flor de Liz Pérez Morales

la cantidad no determina para fines del análisis su discriminación, lo valioso del aporte es que puede resultar significativo en la explicación del objeto de estudio

En la Tabla 1 se muestra la matriz de resultados elaborada a partir de los hallazgos en el grupo de discusión, datos que permiten comprender la perspectiva de los estudiantes sobre los estados del arte como estrategia para plantear problemas de investigación, y de las habilidades investigativas y pensamiento que desarrollan durante el proceso de elaboración del estado del arte, se consideraron las subcategorías definidas al inicio de la investigación, así como la integración de otros ejes encontrados durante la revisión de los datos dados por los sujetos de estudio.

Tabla 1.- Matriz de resultados del grupo de discusión.

\begin{tabular}{|c|c|c|c|}
\hline CATEGORÍAS & SUBCATEGORIAS & RESPUESTAS & TEORÍA \\
\hline Estado del arte & Utilidad/beneficio & $\begin{array}{ll}\text { - } & \text { permite formular ideas } \\
\text { - } & \text { ayuda a delimitar ideas } \\
\text { - } & \text { selección de ideas } \\
\text { - } & \text { comparar investigaciones } \\
\text { - } & \text { desarrollar nuevas ideas } \\
\text { - } & \text { revisar textos } \\
\text { - } & \text { navegar en internet } \\
\text { - } & \text { búsqueda de documentos } \\
\text { - } & \text { no había claridad en la idea } \\
\text { - } & \text { desconoce lo que se hace } \\
\text { - } & \text { dar significado, conocer } \\
\text { - } & \text { búsqueda de documentos } \\
\text { - } & \text { Leer textos una y otra vez } \\
\text { - } & \text { analizar } \\
\text { - } & \text { buscar información para colocarla } \\
& \text { en la matriz }\end{array}$ & $\begin{array}{l}\text { Hacer rastreos por diversos } \\
\text { espacios, } \\
\text { Reunir y organizar conforme al área } \\
\text { de conocimiento, } \\
\text { Análisis sistemático y valoración del } \\
\text { contenido, } \\
\text { Ir tras la huella, } \\
\text { Redacción de síntesis, } \\
\text { Primera etapa que debe } \\
\text { desarrollarse dentro de una } \\
\text { investigación }\end{array}$ \\
\hline Pensamiento & Idea & $\begin{array}{ll}\text { - } & \text { ocurrente } \\
\text { - } & \text { ponerse a pensar en lo que nadie } \\
\text { - } & \text { aprensa } \\
\text { - } & \text { tedioso, aburrido } \\
\text { - } & \text { no existe hasta que yo lo pienso } \\
\text { - } & \text { descubrir algo } \\
\text { - } & \text { piensa y lo aterriza en un escrito } \\
\text { - } & \text { crear propias ideas } \\
\text { - } & \text { llegar a tener propios } \\
\text { - } & \text { conocimientos } \\
\text { crear cosas nuevas }\end{array}$ & $\begin{array}{l}\text { La mente resuelve problemas, } \\
\text { Investigar es pensar, } \\
\text { Hacer rupturas, generar } \\
\text { incertidumbre del pensamiento, } \\
\text { Ejercicio mental, } \\
\text { Recordar, Imaginar, Reflexionar. } \\
\text { Acción de pensar un fenómeno en } \\
\text { particular, sino, sobre todo, como } \\
\text { la lógica con que se piensan los } \\
\text { fenómenos, el mundo y la vida }\end{array}$ \\
\hline & Asociación & $\begin{array}{l}\text { - } \quad \text { poner frente una idea con otra } \\
\text { - } \quad \text { comparar ideas } \\
\text { asociación de las ideas con los } \\
\text { títulos de los textos }\end{array}$ & $\begin{array}{l}\text { Hacer rupturas que impliquen una } \\
\text { crisis de conocimiento. }\end{array}$ \\
\hline Habilidades cognitivas & Autoconocimiento & $\begin{array}{l}\text { - } \\
\text { - } \\
\text { preguntar que se necesita hacer } \\
\text { para elaborar cosas } \\
\text { - } \quad \text { ausencia de ideas, dificultades para } \\
\text { pensar } \\
\text { si pueden descubrir cosas, cuando } \\
\text { les lleve tiempo } \\
\text { requieren de apoyo de un experto } \\
\text { (docente) }\end{array}$ & $\begin{array}{l}\text { Razonar, pensar en los elementos y } \\
\text { en el todo. }\end{array}$ \\
\hline
\end{tabular}


Perspectivas Docentes 64

Investigar-pensar desde los Estados del Arte: Punto de partida Plantear problemas

Research-Thinking from the States of Art: Starting Point Posing Problems

Dra: Rosaura Castillo Guzmán / Dra. Angelica Maria Fabila Echauri / Dra. Flor de Liz Pérez Morales

$\begin{array}{lll} & & \text { define ideas } \\ \text { Aprender a investigar } \quad \text { Aprender } & \text { - } & \text { aprender haciendo } \\ & \text { - } & \text { relación entre conceptos e ideas }\end{array}$

Se forma así mismo, con apoyo de un experto que debe identificar las cualidades del novato.

las universidades y las instituciones deben promover el desarrollo de investigaciones,

Seguir las exigencias básicas del pensamiento riguroso.

La Tabla 2 se incluye la matriz elaborada a partir de los resultados de los cuestionarios, mismos que permiten valorar la perspectiva del estado del arte, las habilidades investigativas y de pensamiento que tienen los estudiantes encuestados.

Tabla 2.- Matriz de resultados de la encuesta.

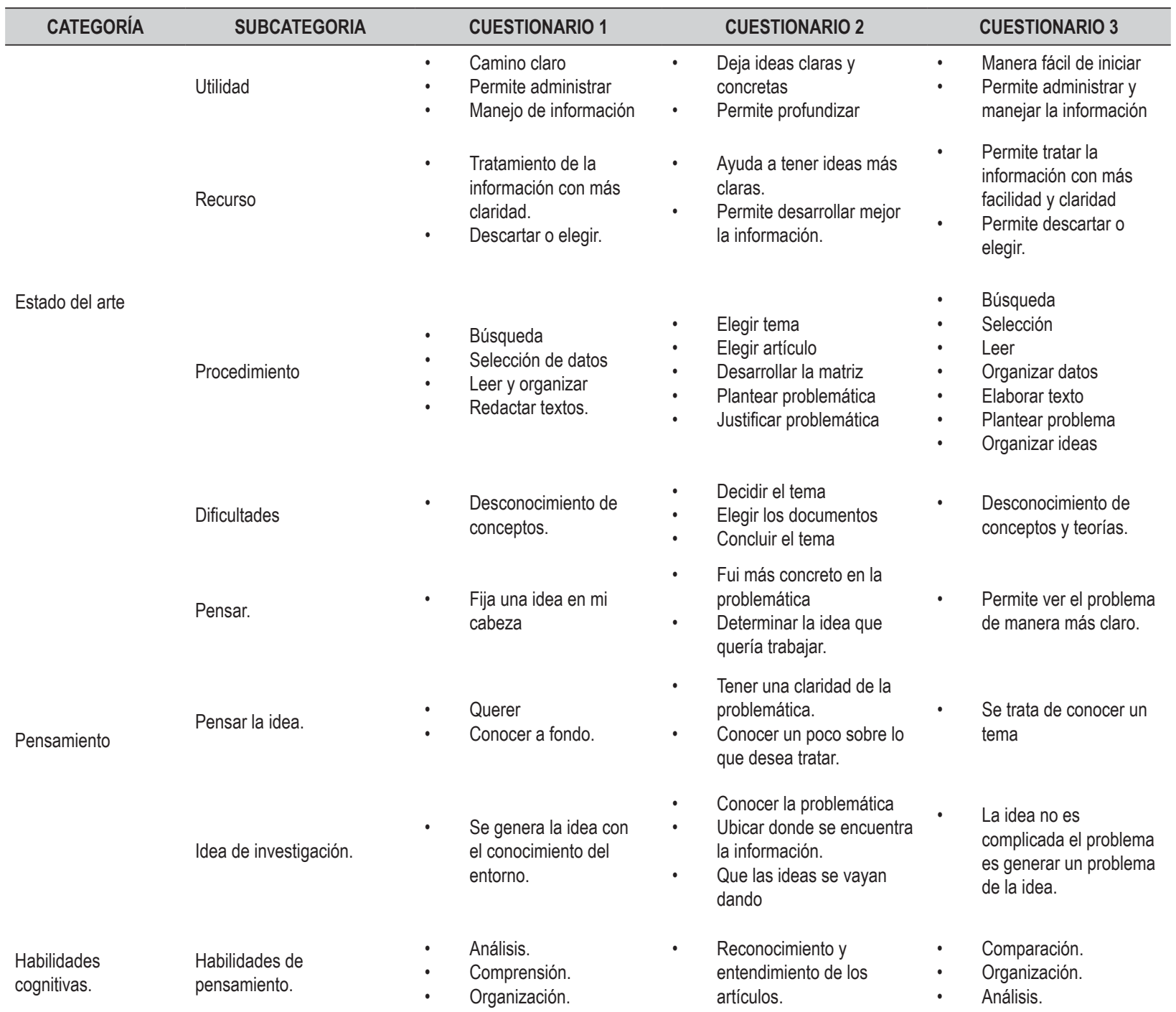




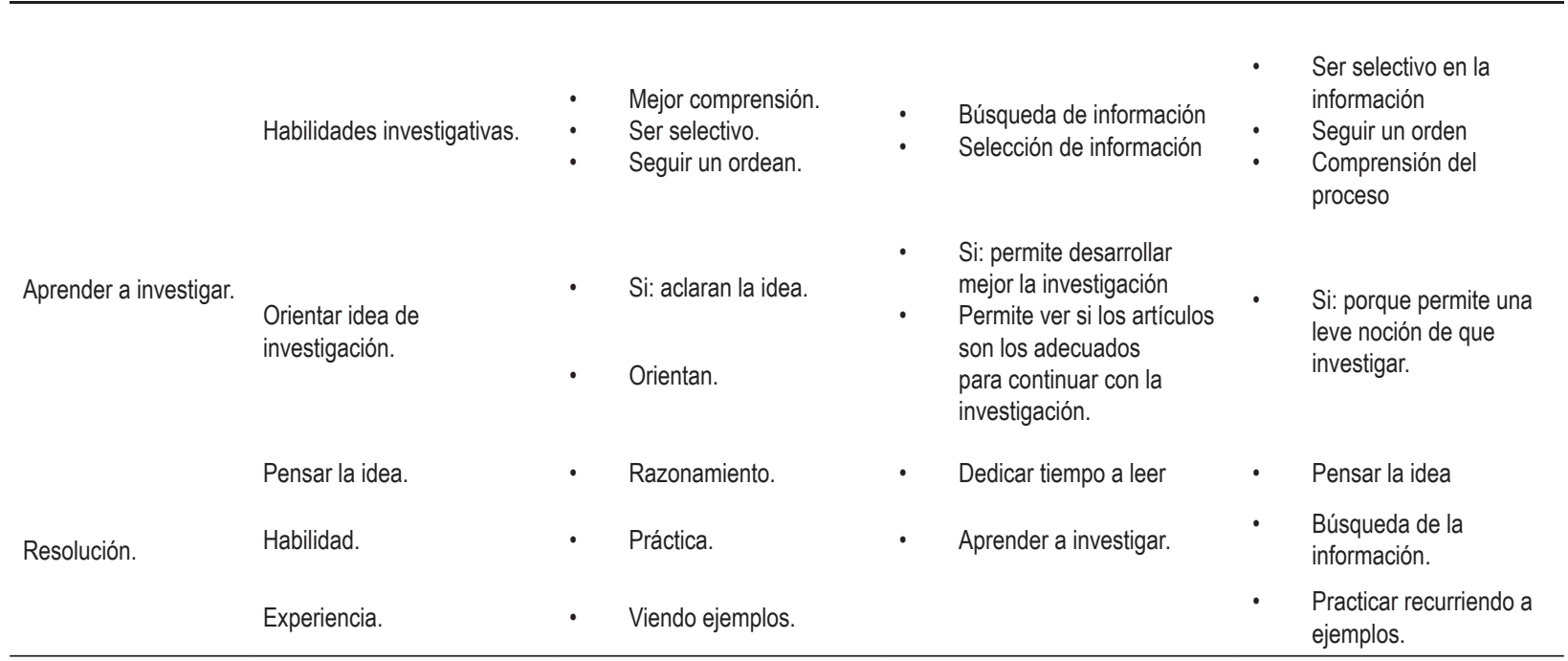

\section{DISCUSIÓN.}

Cada procedimiento de construcción develó que los estudiantes desarrollan algunas habilidades de pensamiento simples, lineal como es el uso de la memoria y de la asociación; el ejercicio de elaboración del estado del arte les generó dudas, pero no solicitan ayuda para resolverlas, lo que les dificultó el desarrollo óptimo del estado del arte.

Al respecto Tesouro (2010), señala que el profesor no tiene que enseñar la materia, sino cómo pensar sobre la materia a aprender. Es deseable que el estudiante aprenda a enfrentarse a la materia con tipos de pensamiento emparentados con lo que hace servir el experto, es decir, el docente.

Otra dificultad identificada a partir de las respuestas fue: el pensar una idea para posteriormente problematizarla; con respecto a las habilidades investigativas, en la búsqueda de los documentos mostraron limitaciones, ya que desconocen los sitios confiables, por ejemplo, en el grupo de discusión estudiantes expresaban que pasan horas navegando en el internet, pero que no podían encontrar un documento que se relacionará con el tema elegido, cuando se les orientaba en la búsqueda situaban páginas confiables, sin embargo la selección de documentos siguió siendo un problema según expresan los estudiantes, poca discriminación, todos los documentos ante sus ojos son valiosos, entonces viene el cuestionamiento de validez 0 de utilidad de lo elegido. El manejo de la matriz para ellos fue difícil en el rubro de categorías, en la identificación de posturas teóricas.
Con respecto a las habilidades de aprendizaje y de cognición se encontró que: aun cuando los estudiantes están en constante uso de la memoria, pocas veces asocian la información de un documento o lo comparan con una idea propia; la organización de la información leída se les dificulta, no logran la asociación entre las categorías o con la idea misma de la investigación que desean emprender, lo que los hace ver ante los ojos del "experto" como verdaderos novatos.

Lo anterior, nos hace reconocer que el mundo que miran los estudiantes, no es el mismo mundo que miran los docentes, sin embargo existen planteamientos que el mundo actual debe ser comprendido tal y como se nos presenta, al respecto Morín (1994) plantea que la complejidad del mundo actual requiere de un pensamiento que trate de ser coherente con la realidad; no se debe pensar en fenómenos complejos con principios simples pues una inteligencia incapaz de encarar el contexto y el complejo global se vuelven ciegos, inconscientes e irresponsables.

La percepción que se tiene del uso del estado del arte como estrategia que ayuda al novato en el aprendizaje de aprender a pensar, es positiva, los novatos interactúan con la matriz que les permite una organización de las ideas leídas, sin embargo se pudo identificar en el proceso que no sólo es actitud de querer aprender, es importante el desarrollo del pensamiento crítico, la generación de ideas y el desarrollo de habilidades investigativas, con esta combinación de acciones y habilidades los estados del arte pueden posibilitar al estudiante a acceder a otras formas de pensar la investigación. 
Perspectivas Docentes 64

Investigar-pensar desde los Estados del Arte: Punto de partida Plantear problemas

Research-Thinking from the States of Art: Starting Point Posing Problems

Dra: Rosaura Castillo Guzmán / Dra. Angelica Maria Fabila Echauri / Dra. Flor de Liz Pérez Morales

\section{CONCLUSIONES}

Lograr que el conocimiento de los aprendices-novatos que poseen una estructura cognitiva, sea aprovechada con el uso de estrategias para promover el aprender a aprender, esto hará posible en el estudiante la propia identificación de sus procesos de aprender y cuando los expertos realicen de igual manera ese reconocimiento, el proceso didáctico podría garantizar una mejor formación del aprendiz-novato y una enseñanza acorde a las características cognitivas, de pensamiento del estudiante.

Bien podría el docente, partir de la idea expuesta por Edgar Morín sobre el desarrollar el pensamiento complejo en los estudiantes, lo que les permitirá dar orden al pensamiento, llevarlo al diálogo consigo mismo, de replantearse situaciones, es decir, intentar comunicarse y enlazar todas las dimensiones de lo humano.

La cultura de investigación no se crea en el vacío, ni tampoco opera por decreto o por propuesta curricular como equivocadamente algunos docentes creemos. Como base en dicha cultura deben existir fundamentos y condiciones académicas y administrativas, que sustenten adecuadamente el quehacer investigativo. Solo así es posible hacer sostenible en el tiempo, la construcción de una cultura investigativa institucional de raíces firmes y de largo aliento, capaz de generar impactos duraderos en el pensar y hacer de las distintas generaciones de aprendices y novatos que se forman a partir de los programas académicos que la institución aplica.

La problemática vivida por los estudiantes al momento de construir el estado del arte y develar un planteamiento del problema, se considera como un reto para quien enseña, ya que su responsabilidad pedagógica, de experto es guiar al estudiante-novato al desarrollo de la investigación, al respecto Blanco (2010) puntualiza que investigar es una actividad que puede ser practicada por cuanto sigan las exigencias básicas del pensamiento riguroso; ser investigador es, antes que nada, ser estudioso de algo, alguien que piensa.

Algunos hallazgos y rupturas encontradas en esta exploración se dirigen a señalar que las universidades son responsables de la formación de investigadores y en esa materia han hecho intentos valiosos, pero que no han sido suficientes; estos momentos de incertidumbre requieren de cambios vertiginosos en su estructura socio-política y económica, que convaliden al conocimiento como una de las mercancías más preciadas y ante ese escenario se ofrezcan propuesta educativas acordes.
La investigación es una actividad sustantiva en las universidades y se apuesta a esta actividad para generar conocimientos pertinentes que hagan posible un mejor mundo. Las universidades deben pensar en el futuro de las profesiones, en este sentido Morín (1994) sugiere una transformación en el paradigma de la formación, donde el pensamiento complejo articulado con las ideas de la transdisciplinariedad busque reconocer un conocimiento desde la totalidad de la vida.

Ante el panorama de incertidumbre, la noción de enseñanza y aprendizaje ya no puede ser concebida como una acción pedagógica cerrada, dadas las características del mundo actual se requiere formar sujetos que comprendan las transformaciones de su contexto, para ello las instituciones deben apostar a propuestas educativas bajo el paradigma de la complejidad.

Aprender y enseñar a investigar a través de los estados del arte, ha dejado una experiencia de aplicación como estrategia didáctica para enseñar a pensar una idea y a partir de ahí iniciar con el planteamiento del problema; un planteamiento pensado y contrastado desde la experiencia del aprendiz-novato.

Se recupera las dificultades que viven o enfrentan para darle forma a la idea, ya que esto debe permitir al docente 0 al experto a buscar estrategias que acompañen la enseñanza a través de los estados del arte, y que el pensar es una actividad que encuentra dificultades cuando se les pide dar orden al pensamiento, se convierta en una tarea menos compleja para el aprendiz-novato.

Ambas posturas, tanto el que enseña como el que aprende, viven sus propias dificultades frente a los estados del arte, sin embargo, cuando se crean las condiciones pertinentes puede ser una actividad sugerida y guiada por expertos, la enseñanza para aprender a investigar puede encontrar terreno fértil al reconocer las habilidades de pensamiento y las formas cómo aprende el estudiante a investigar. 


\section{REFERENCIAS}

Aguirre. M. (2001) Rostros Históricos de la Educación. México: Fondo de Cultura Económica.

Babbie, E. (2000). Fundamento de la Investigación social. Barcelona: Ediciones Paraninfo.

Blanco, G.N. (2010). La investigación en el ámbito del curriculum y como método para su desarrollo. En saberes e incertidumbres sobre el curriculum. Madrid: Ediciones Morata.

Consejo Mexicano de Investigación Educativa (2011). Estado del Arte. Boletín 1. Consultado en http://issuu.com/maye/docs/ estado_del_artefinal septiembre 2011.

Cabrera, A. J. (2010). Edgar Morin. Recuperado el 23 de julio de 2013, de Edgar Morin: www.sitiomorin.com.

Cabrera, A.J. (2014). Edgar Morín y el Pensamiento de la Complejidad. Ciencias de la Educación. México. Edit. Trillas

Castañeda, Z.D.I (2004). Estado del arte en aprendizaje organizacional, a partir de las investigaciones realizadas en facultades de psicología, ingeniería industrial y administración de empresas en Bogotá, entre los años 1992 y 2002. Recuperado de http://portalweb.ucatolica.edu.co/easyWeb2/ acta/pdfs/n11/art_3_acta_11.pdf

Delgado, J.M. y Gutiérrez, J. (eds.) (1998). Métodos y técnicas cualitativas de Investigación social. Madrid: Editorial Síntesis

Herrera, A., \& Didrikson, A. (2012). Curriculum. México: Trillas.

Herrera, M. A. (2007). Guía de autoevaluación de proyectos de investigación educativa. Facultad de Estudios Superiores Zaragoza.

López R, N. García F (2012). El proyector Integrador. Estrategia didáctica para la formación de competencias desde la perspectiva del enfoque socioformativo. Edit, PaisGrafá

Martínez, M.M. El paradigma emergente. Hacia una nueva teoría de la racionalidad científica. México, Edit. Trillas
Medina, C.M (2008). Un estado del arte sobre los textos infantiles ilustrados. México. Correo del maestro.

Mejía R. Sandoval S. (2003). Tras las Vetas de la investigación cualitativa Perspectivas y acercamiento desde la práctica. ITESO, Jalisco, México.

Morín, E. (1999). Los 7 Saberes necesarios para la educación del futuro, Francia: Santillana.

Morin, E. (1994). Introducción al pensamiento complejo. Sitio oficial Edgar Moriin. (Recuperado el 23 de enero de 2014), de sitio oficial Edgar Morin: http://www.sitiooficialedgarmorin.com. $\mathrm{mx}$

Nickerson, R.S. Perkin y Smith (1998). Enseñar a pensar. Aspectos de la aptitud intelectual, Barcelona, España. Paidós

Moreno, B. (2011). La formación de investigadores como elementos para la consolidación de la investigación en la universidad. Educación Superior, 59-78.

Núñez, M. A., Arévalo, A. y Ávalos, B. (2012). Profesionalización docente: ¿es posible un camino de convergencia para expertos y novatos? Revista Electrónica de Investigación Educativa, 14(2), 10-24. [Fecha de consulta: 15/noviembre/2013]. http:// redie.uabc.mx/vol14no2/contenido-nunezetal.html

Jiménez, J.M; Moreno B, M.G; Ortiz L, V. (2011). Culturas de los estudiantes de investigación en programas de doctorado en educación. Reflejos de un campo en definición. Revista Mexicana de Investigación Educativa, Julio-Septiembre, 919933.

Palva, C. A. J. (2014). Edgar Morín y el Pensamiento de la Complejidad. Ciencias de la Educación. http://servicio.bc.uc. edu.ve/educacion/revista/a4n23/23-14.pdf

Pozo, J.I, Monereo, C. (1999). El aprendizaje estratégico. Enseñar a aprender desde el curriculum. Madrid, España. Aula XXI Santillana

Ramos, J. Alba F (2011). Para la elaboración de estados del conocimiento de la investigación educativa, 2002-2011. http:// www.comie.org.mx/congreso/memoriaelectronica/v10/pdf/ area_tematica_11/ponencias/0494-F.pdf 
Perspectivas Docentes 64

Investigar-pensar desde los Estados del Arte: Punto de partida Plantear problemas

Research-Thinking from the States of Art: Starting Point Posing Problems

Dra: Rosaura Castillo Guzmán / Dra. Angelica Maria Fabila Echauri / Dra. Flor de Liz Pérez Morales

Rojas, S.P (2007). El estado del arte como estrategia de formación en la investigación. Universidad Pedagógica y Tecnológica de Colombia. http://metadirectorio.org/ bitstream/10983/504/1/Stud_2-3_A01_ROJAS-ROJAS.pdf

Rodríguez G, G, J. Gil Flores y E. García Jiménez (1996). Metodología de la investigación cualitativa. Málaga: Ediciones Aljibe.

Sandín E. M.P. (2004): Investigación cualitativa en educación. Fundamentos y tradiciones. [Fecha de consulta: 17/noviembre/2014]. http://www.saber.ula.ve/ handle/123456789/24057

Sternberg, R.J, Louise (1996). Enseñar a pensar. Madrid, España. Aula XXI Santillana.
Strauss, A. Corbín J (2002). Bases de la Investigación cualitativa. Técnicas y procedimientos para desarrollar la teoría fundamentada. Colombia. Publications, Inc..

Tesouro Cid, Montse (2006). Enseñar a aprender a pensar en los centros educativos, incluso en las actividades de evaluación. REIFOP, 9 (1). (Enlace web: http://www.aufop.com/ aufop/home/ - Consultada en fecha (27-septiembre- 2011)

Tedesco, J. (2003). "Los pilares de la educación del futuro". En: Debates de educación (2003: Barcelona) [ponencia en línea]. Fundación Jaume Bofill; UOC. [Fecha de consulta: 30/ octubre/2013]. <http://www.uoc.edu/dt/20367/index.html> 